\title{
Comprehensive cluster-theory analysis of the magnetic structures and excitations in
} $\mathrm{CoCl} 2 \cdot 2 \mathrm{H} 2 \mathrm{O}$

Jensen, Jens; Larsen, Jacob; Hansen, Ursula B.

Published in:

Physical Review B

Link to article, DOI:

10.1103/PhysRevB.97.024423

Publication date:

2018

Document Version

Publisher's PDF, also known as Version of record

Link back to DTU Orbit

Citation (APA):

Jensen, J., Larsen, J., \& Hansen, U. B. (2018). Comprehensive cluster-theory analysis of the magnetic structures and excitations in $\mathrm{CoCl}_{2} \cdot 2 \mathrm{H}_{2} \mathrm{O}$. Physical Review B, 97(2), [024423].

https://doi.org/10.1103/PhysRevB.97.024423

\section{General rights}

Copyright and moral rights for the publications made accessible in the public portal are retained by the authors and/or other copyright owners and it is a condition of accessing publications that users recognise and abide by the legal requirements associated with these rights.

- Users may download and print one copy of any publication from the public portal for the purpose of private study or research.

- You may not further distribute the material or use it for any profit-making activity or commercial gain

- You may freely distribute the URL identifying the publication in the public portal

If you believe that this document breaches copyright please contact us providing details, and we will remove access to the work immediately and investigate your claim. 


\title{
Comprehensive cluster-theory analysis of the magnetic structures and excitations in $\mathrm{CoCl}_{2} \cdot 2 \mathrm{H}_{2} \mathrm{O}$
}

\author{
Jens Jensen, ${ }^{1}$ Jacob Larsen, ${ }^{2}$ and Ursula B. Hansen ${ }^{1}$ \\ ${ }^{1}$ Niels Bohr Institute, Universitetsparken 5, 2100 Copenhagen, Denmark \\ ${ }^{2}$ Department of Physics, Technical University of Denmark, 2800 Kgs. Lyngby, Denmark
}

(Received 12 October 2017; revised manuscript received 22 December 2017; published 22 January 2018)

\begin{abstract}
The magnetic properties of $\mathrm{CoCl}_{2} \cdot 2 \mathrm{H}_{2} \mathrm{O}$ are analyzed in the mean-field/random-phase approximation using a basis of clusters with four spins along the $c$-axis chains of Co ions. The model gives a unifying account of the bulk properties, the spin waves, and the higher-order cluster-spin excitations. The theory describes accurately the neutron scattering measurements of the excited doublet of the $S=3 / 2 \mathrm{Co}^{++}$ions in both the antiferromagnetic and the paramagnetic phases. The theory has been applied by Larsen et al. [Phys. Rev. B 96, 174424 (2017)] for analyzing the quantum phase transition at a transverse field of $160 \mathrm{kOe}$ and is found to agree closely with their observations.
\end{abstract}

DOI: 10.1103/PhysRevB.97.024423

\section{INTRODUCTION}

$\mathrm{CoCl}_{2} \cdot 2 \mathrm{H}_{2} \mathrm{O}$ has previously been considered to be a fair representation for a one-dimensional Ising system, however, as shown by Larsen et al. [1], the quantum phase transition of the system in a transverse field is characterized by critical exponents equal to those derived for a three-dimensional, meanfield system. Here, we are going to present a comprehensive and realistic model for the magnetic properties of $\mathrm{CoCl}_{2} \cdot 2 \mathrm{H}_{2} \mathrm{O}$. The theory is based on clusters with four neighboring spins along the $c$-axis chains, and includes the effects of the excited doublet of the single Co spins.

The question whether Bloch oscillations may exist in magnetic systems and, in particular, in the Ising-like chain system $\mathrm{CoCl}_{2} \cdot 2 \mathrm{H}_{2} \mathrm{O}$ has been raised several times in the literature. The most recent and most realistic analysis is the one presented by Shinkevich and Syljåsen [2]. Prior to their work, the possibility of observing Bloch oscillations in anisotropic spin- $\frac{1}{2}$ chains was discussed by Kyriakidis and Loss [3]. Shinkevich and Syljåsen argue that the longitudinal field deriving from the interactions between spins on neighboring chains invalidates the single domain-wall picture of Kyriakidis and Loss by creating bound pairs of domain walls [2]. The dynamic of the bound pairs of walls, in the antiferromagnetic phase of $\mathrm{CoCl}_{2} \cdot 2 \mathrm{H}_{2} \mathrm{O}$, becomes equivalent to that shown by the spin waves and additional magnon bound states or, more specifically, by the spin reversal of clusters of spins consisting of 1,2 , 3 , or more spins along the $c$-axis chains. These excitations were observed in $\mathrm{CoCl}_{2} \cdot 2 \mathrm{H}_{2} \mathrm{O}$ by means of far-infrared transmission measurements, and explained in all details, in a pioneering work by Torrance and Tinkham $[4,5]$.

The present cluster theory accounts both for the spin waves and for the spin cluster excitations with the same set of parameters, which parameters are fully consistent with the paramagnetic susceptibility tensor and give a fair account of the low-temperature magnetization curves. The predictions made by this theory compare in most practical details with those made by Shinkevich and Syljåsen [2], and thus favors their basic argument that the dynamics of this chain system derives from bound pairs of domain walls. Of major importance for the development of the model is our investigation of the excited doublet by a neutron time-of-flight experiment. We also present measurements of the spin waves, where the moments are rotated away from the easy axis by applying a field of up to $170 \mathrm{kOe}$, with most focus on the soft mode behavior shown by the spin waves close to the quantum-phase transition, where the antiferromagnetic ordered is destroyed $[1,6]$.

The crystal structure of $\mathrm{CoCl}_{2} \cdot 2 \mathrm{H}_{2} \mathrm{O}$, or of the deuterated version $\mathrm{CoCl}_{2} \cdot 2 \mathrm{D}_{2} \mathrm{O}$ applied in neutron scattering experiments [7], is monoclinic and belongs to the space group $C 2 / \mathrm{m}$. The structure consists of chains of $\mathrm{Co}^{++}$ions along the $c$ axis. The different chains are separated by the water molecules. There are two Co sites with identical surroundings per monoclinic unit cell, at $(0,0,0)$ and $\left(\frac{1}{2}, \frac{1}{2}, 0\right)$. More details and a figure illustrating the crystal structure may be found in our preceding publication [1]. A quite complete model for the magnetic properties of the bulk system has been established by Narath [8,9]. Below the Néel temperature $T_{N} \simeq 17.5 \mathrm{~K}$, the chains are ferromagnetic ordered, but the moments on the chain through $\left(\frac{1}{2}, \frac{1}{2}, 0\right)$ are antiparallel to the moments belonging to the chain through $(0,0,0)$. The system is strongly anisotropic and may in some respect be classified as an Ising system, but it is also clear that the dynamics, we are going to discuss, would not be there if not for the interactions between the $x$ and $y$ components of the spins. The spins are strongly coupled along the chains implying that it shows some one-dimensional-like properties, but the ratio between the inter- and intrachain couplings is not really small but about 0.4 . The system bears resemblance with another Co compound $\mathrm{CoNb}_{2} \mathrm{O}_{6}$, but in this system the ratio is much smaller, about 0.02 , and its one-dimensional character is more pronounced [10].

\section{THE MODELING OF THE CO SPIN SYSTEM}

Co compounds are, generally, strongly anisotropic systems and may show Ising-like behavior in low-symmetry surroundings. The $\mathrm{CoO}$ compound has recently been investigated by Cowley et al. by high-energy scattering of neutrons [11], and one of their conclusions is, that although the anisotropy 
TABLE I. The single-ion parameters in units of meV.

\begin{tabular}{ccccc}
\hline \hline$E_{P}$ & $\zeta$ & $B_{4}^{0}$ & $B_{2}^{0}$ & $B_{2}^{2}$ \\
\hline 1400 & 21.82 & -2.6 & -2.9 & -3.3 \\
\hline
\end{tabular}

is strong, the crystal-field effects on the Co ions are still within the weak coupling limit. Accepting this to be valid also for the $\mathrm{Co}^{++}$ions in $\mathrm{CoCl}_{2} \cdot 2 \mathrm{H}_{2} \mathrm{O}$, we have used the approach of Abragam and Pryce [12], corresponding to the intermediate ligand field theory of Abragam and Bleaney [13], for constructing a realistic model for the lowest levels in the $\mathrm{Co}^{++}$ions. The lowest term in the $3 d^{7}$ configuration of the free $\mathrm{Co}^{++}$ion is the ${ }^{4} F$ term, while the energy difference to the next lowest one, ${ }^{4} P$, is $E_{P} \simeq 1.7 \mathrm{eV}$. The Hamiltonian for the single $\mathrm{Co}^{++}$ion, when being in the ground state term with $S=3 / 2$ and $L=3$, is assumed to be

$$
\begin{aligned}
\mathcal{H}_{\mathrm{I}}= & B_{4}^{0}\left[O_{4}^{0}(L)+5 O_{4}^{4}(L)\right]+B_{2}^{0} O_{2}^{0}(L)+B_{2}^{2} O_{2}^{2}(L) \\
& -\zeta \vec{L} \cdot \vec{S}-\mu_{B} \vec{H} \cdot(\vec{L}+2 \vec{S})
\end{aligned}
$$

expressed in terms of Stevens operators [13-15], e.g., $O_{2}^{0}(L)=$ $3 L_{z}^{2}-\vec{L} \cdot \vec{L}$. The effects due to the next lowest term ${ }^{4} P$ with $L=1$ are included in the model calculations, which requires the Stevens operators in Eq. (1) to be replaced by the corresponding tensor operators proportional to the Racah operators. The matrix elements of these tensor operators for the combined ${ }^{4} F-{ }^{4} P$ system are given (indirectly) in Ref. [12]. For the sake of simplicity, the parametrization of Eq. (1) has been kept. In particular, the $B_{\ell}^{m}$ coefficients of the Stevens Hamiltonian, giving the splitting of the $L=3$ ground multiplet in the limit of crystal field small with respect to $E_{P}$, are used. The Racahs parameters useful for the solution of the twomultiplet problem are simply related to the $B_{\ell}^{m}$ coefficients by numerical factors [14]. Although the ${ }^{4} P$ term is lying far above the ground state, it is important for determining the splitting between the two lowest doublets, and it also have perceptible effects on the wave functions of the ground-state doublet. Any other terms than these two lowest ones are neglected. The first crystal-field term in Eq. (1), $B_{4}^{0}$, has cubic symmetry and accounts for the major bulk effects of the crystal field without making any distinction between an $x, y$, or $z$ direction. Defining the main axes of the anisotropy tensor so that $z$ is the easiest axis, i.e., the $b$ axis, and $y$ the hardest one [1], it may, effectively, be accounted for by the two quadrupolar terms in Eq. (1) with $B_{2}^{0}<0$ and $B_{2}^{2}<0$. The fitting parameters are the three crystal-field parameters and $E_{P}$, whereas the spin-orbit coupling $\zeta$ has been fixed to the value assumed by Narath $[9,12]$.

The final values of these parameters determining the low energy properties of the single $\mathrm{Co}^{++}$ions are given in Table $\mathrm{I}$. The spectrum derived from the parameters in the table consists of two Kramers doublets separated by $18.8 \mathrm{meV}$, whereas the next excited doublet lies at about $100 \mathrm{meV}$ above the ground state. Considering that the energy changes due to the interaction with other Co moments or to an applied field are of the order of a few meV, the influences due to the third or higher-lying doublets are weak and may be neglected in most cases. This leaves us with a two-doublet system, which may be described
TABLE II. The effective $g$ factors for the ground state doublet, as defined by Narath [9], and determined by the matrix elements within the doublet.

\begin{tabular}{lllllll}
\hline \hline & $g_{S}^{x}$ & $g_{L}^{x}$ & $g_{S}^{y}$ & $g_{L}^{y}$ & $g_{S}^{z}$ & $g_{L}^{z}$ \\
\hline Table I & 2.817 & 0.692 & 1.879 & 0.062 & 4.827 & 1.775 \\
Narath[9] & 2.67 & 0.68 & 1.83 & 0.04 & 4.95 & 1.82 \\
\hline \hline
\end{tabular}

in terms of an effective $S$ (eff) $=3 / 2$ model. Our model for the $\mathrm{Co}^{++}$ions is similar to the one developed by Narath [9]. He placed the excited doublet state at the slightly lower energy of $\sim 16.9 \mathrm{meV}$, but the properties of the ground state in his model are close to that determined by the parameters in Table I. A comparison between the results of the two models for the effective $g$ factors of the ground state doublet, as defined by Narath [9], is shown in Table II. The total effective $g$ factor for the $\xi$ axis is the sum of the spin and orbital $g$ factors: $g^{\xi}=g_{S}^{\xi}+$ $g_{L}^{\xi}$. We have to stress that the parameters in Table I are effective ones, their values are not necessarily close to the right ones. For instance, keeping $B_{2}^{0}$ unchanged and introducing $B_{4}^{2} O_{4}^{2}(L)$ in Eq. (1), the values of $\left(E_{P}, B_{4}^{0}, B_{2}^{2}, B_{4}^{2}\right)$ may be replaced by sets like $(1800,-2.5,0,-0.9) \mathrm{meV}$ or $(2000,-3.7,-3.3,0) \mathrm{meV}$ with nearly no consequences for the $g$ factors in Table II, or the other calculated results reported in this paper.

The interactions between the Co moments are assumed to be due alone to Heisenberg exchange between the Co spins:

$$
\mathcal{H}_{\mathrm{II}}=-\frac{1}{2} \sum_{i, j} J(i, j) \vec{S}(i) \cdot \vec{S}(j) .
$$

The exchange parameters, which in combination with the single ion parameters in Table I define the final model, are given in Table III. The interaction $J_{0}$ between nearest neighbors along the $c$ axis is an order of magnitude larger than any other exchange parameter. The simple mean-field (MF) and randomphase (RPA) approximations, where the basis is the single spins, treat all interactions on equal footing and may not be trusted in this case. One way to improve these approximations is to use clusters of strongly coupled spins as basis instead of the single spins. This leads to clear improvements in the cases of dimer, trimer, or tetramer systems (see, for instance, Refs. $[16,17])$. In the present case of chains of strongly coupled spins, we have considered clusters consisting of 2 , 3 , or $4 \mathrm{Co}^{++}$neighboring ions along the $c$-axis chains. The Hamiltonian for one cluster including all interactions between the constituents and the mean fields from the surrounding clusters, is diagonalized numerically, where the mean fields from the neighboring, equivalent clusters are being determined self-consistently by iteration. After that has been done, we may

TABLE III. The two-ion exchange parameters. The position vectors $\vec{r}_{i}-\vec{r}_{j}$ in $J(i, j)=J\left(\vec{r}_{i}-\vec{r}_{j}\right)$ are given in terms of the lattice vectors $\vec{a}, \vec{b}$, and $\vec{c}$.

\begin{tabular}{cccccc}
\hline \hline$J(i, j)$ & $J_{0}$ & $J_{0}^{\prime}$ & $J_{1}$ & $J_{1}^{\prime}$ & $J_{2}$ \\
\hline$\vec{r}_{i}-\vec{r}_{j}$ & $\pm \vec{c}$ & $\pm 2 \vec{c}$ & $\pm \frac{1}{2} \vec{a} \pm \frac{1}{2} \vec{b}$ & $\pm\left(\frac{1}{2} \vec{a}+\vec{c}\right) \pm \frac{1}{2} \vec{b}$ & $\pm \vec{a}$ \\
{$[\mathrm{mev}]$} & 0.66 & -0.08 & -0.045 & -0.085 & -0.025 \\
\hline \hline
\end{tabular}


calculate the generalized susceptibility tensor for one cluster and from this the cluster RPA response functions [15-18]. The division of the chains in sections with, for instance, four ions in each, introduces an extra, artificial periodicity of the system. This may result in spurious peaks in the correlation functions, but this is not a serious problem, since the extra peaks are weak and are easily identified in most cases.

The previous analyses of the magnetic excitations in $\mathrm{CoCl}_{2} \cdot 2 \mathrm{H}_{2} \mathrm{O}$ apply an approximation, where the excited doublet is neglected [5,19-21]. The relation between the exchange parameters of the $S($ eff $)=1 / 2$ model used in these papers and the ones in Eq. (1) or Table III, is $(\xi=x, y$, or $z)$

$$
\begin{aligned}
& \mathcal{H}_{\mathrm{II}}=-\sum_{i, j} \sum_{\xi=x, y, z} J^{\xi}(i, j) s_{\xi}(i) s_{\xi}(j), \quad s=\frac{1}{2}, \\
& J^{\xi}(i, j)=\frac{1}{2}\left(\frac{g_{S}^{\xi}}{2}\right)^{2} J(i, j)
\end{aligned}
$$

in terms of the spin $g$ factors in Table II. The extra factor $1 / 2$ is due to the factor $1 / 2$ in front of the sum in Eq. (1), which is left out in the definition of the exchange parameters in Refs. [5,19-21]. Two important parameters characterizing the anisotropy of the $S($ eff $)=1 / 2$ model are

$$
\begin{aligned}
j^{\perp} & =\left[J^{x}(i, j)+J^{y}(i, j)\right] / 2 J^{z}(i, j)=0.246(0.214), \\
j^{a} & =\left[J^{x}(i, j)-J^{y}(i, j)\right] / 2 J^{z}(i, j)=0.095(0.077),
\end{aligned}
$$

where the numbers are determined by the $g_{S}^{\xi}$ values in Table II with Narath's results given in parentheses. In the Heisenberg spin model assumed here, Eq. (2), the two parameters are independent of the pair of spins $(i, j)$ considered.

Preliminary analyses of $\mathrm{CoCl}_{2} \cdot 2 \mathrm{H}_{2} \mathrm{O}$ using the cluster theory, but with slightly different values for the parameters, have been presented by Jacob Larsen in his Master and Ph.D. theses [22]. In the next sections, we are going to compare the predictions of the present model with experiments.

\section{SINGLE-ION PROPERTIES AND MAGNETIC ANISOTROPY}

An important property of the $\mathrm{Co}^{++}$ion is the position of the lowest excited doublet. Model calculations were able to predict about the right energy, and that the scattering intensity of this level should be somewhat larger than that shown by the spin waves in the antiferromagnetic phase. The higher lying doublet excitation was measured at the direct time-of-flight spectrometer MARI (ISIS, Rutherford Appleton Laboratories, UK). The experimental details are presented in Ref. [1], and the results are summarized in Fig. 1. Because of the $Q^{2}$ dependence of the phonon cross section, in contrast to a decreasing magnetic intensity for increasing $Q$, the phonons appear with much increasing weight, when the radius of integration in $Q$ space is changed from 4 to $9 \AA^{-1}$. This implies that we may assign the scattering accounted for by the calculated blue line, which scattering is more or less unaffected by the change of integration interval, to be magnetic, whereas the remaining part, which is roughly changed by a factor of 2 by the change in integration volume, is due to the phonons.

To leading order, the powder averaging over $Q$ space leads to a scattering peak at an energy derived for an excitation in
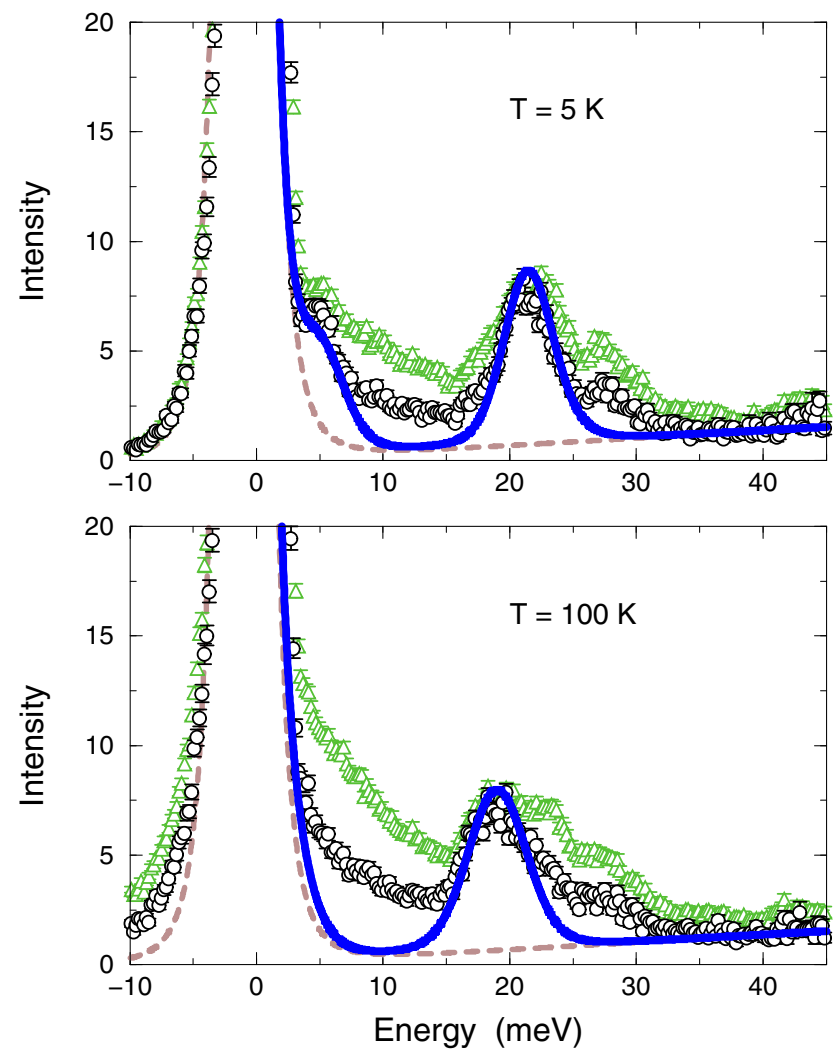

FIG. 1. Data from the MARI experiment showing the $Q$ integrated intensity vs energy at $5 \mathrm{~K}$ and $100 \mathrm{~K}$. The black circles are the results obtained when integrating the scattering intensity with respect to $Q$ between 0 and $4 \AA^{-1}$, whereas the green triangles are the results obtained, when integrating from 0 to $9 \AA^{-1}$. The blue solid lines are the calculated results obtained using a cluster basis of three spins along the Co chains, when assuming the background scattering shown by the dashed brown lines.

$Q$ space, where the exchange interaction vanishes, and with a linewidth determined by the band width of the excitations convoluted with the experimental resolution function. The use of this approximation leads to the results shown by the solid blue lines in Fig. 1. Both the intensity scale factor and the resolution function, a Gaussian with $\sigma=1.6 \mathrm{meV}$, are assumed to be the same at 5 and at $100 \mathrm{~K}$. The results at $100 \mathrm{~K}$ show clearly that the excited doublet is placed at about $19 \mathrm{meV}$ above the ground state doublet in the paramagnetic phase. In agreement with the calculations, the peak is shifted up in energy by about $2.5 \mathrm{meV}$ in the antiferromagnetic phase at $5 \mathrm{~K}$. It is also worth to notice that the peak is broader, both experimentally and theoretically, at $100 \mathrm{~K}$ compared to the result at $5 \mathrm{~K}$. The calculated extra broadening at $100 \mathrm{~K}$ is genuine and derives from that the ground state doublets of the single spins are modified into a broad distribution of states due to the internal interactions within the spin clusters.

The experimental susceptibility components in the paramagnetic phase are compared with the results derived from our model using three different approximations in Fig. 2. The three approximations all apply, eventually, the MF approximation, but start out from three different bases. The version based on the 1D chain is presented in the Appendix. The Néel temperatures 


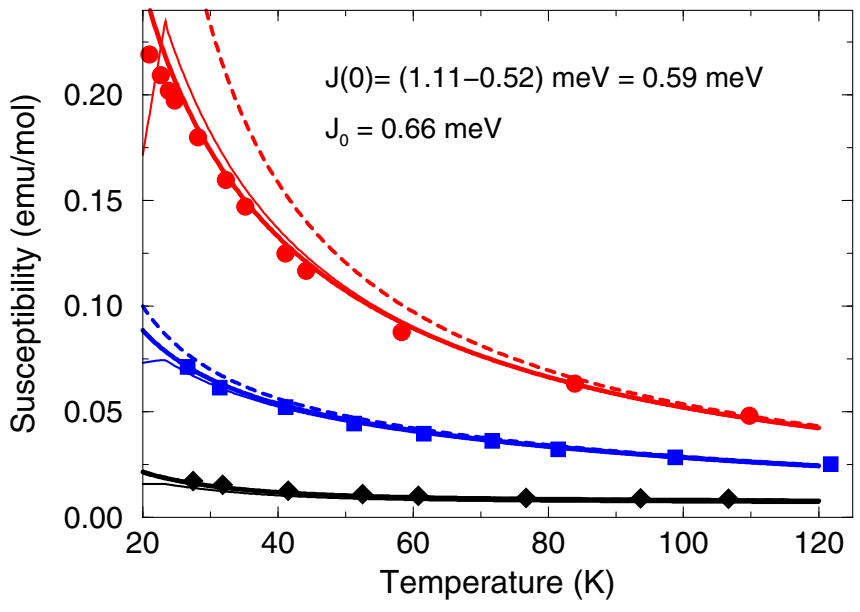

FIG. 2. The red circles, the blue squares, and the black diamonds show, respectively, the experimental results for the $z z$, the $x x$, and the $y y$ component of the susceptibility tensor in the paramagnetic phase of $\mathrm{CoCl}_{2} \cdot 2 \mathrm{H}_{2} \mathrm{O}$ measured by Narath [9]. The heavy solid lines with the same colors as the corresponding experimental points, are the results obtained by a perturbative MF modification of the $1 \mathrm{D}$ chain susceptibilities. The thin solid lines are the MF results obtained when using the four-spin chain clusters as basis, whereas the dashed lines are the simple MF results assuming a single spin basis.

predicted are $29.8,23.4$, and $19.0 \mathrm{~K}$ for, respectively, the single spin, four-spin cluster, and the 1D-chain approximation. The smaller the difference is between the predicted one and the experimental value $T_{N} \simeq 17.5 \mathrm{~K}[7,8]$, the better is the approximation, and we may conclude that the use of the four-spin chain cluster instead of the single spin basis leads to clear improvements. The fit shown in Fig. 2 is in closer agreement with experiments than that obtained by Narath (see Fig. 12 in Ref. [9]) implying that the present $g$ factors in Table II should be more trustworthy than those derived by Narath from his analysis of the susceptibility data.

Figure 3 shows the magnetization curves along the three main axes measured by Mollymoto et al. at $1.3 \mathrm{~K}$ in the antiferromagnetic phase [23]. The calculated results are obtained using the 4-spin chain-cluster basis with $S($ eff $)=3 / 2$. MF calculations using the single spins as basis show that the higher lying levels neglecting in the $S$ (eff) $=3 / 2$ model are of some importance at large values of the field and lead to a $1 \%-2 \%$ increase of the moments at maximum field along $z$ or $x$, but about a $10 \%$ increase in the $y$-axis case. These extra contributions are added to the moments derived in the $S($ eff $)=3 / 2$ cluster model in the final results shown in Fig. 3. In the case where the field is applied along the easy $b$ axis, the experiments show that there is an intermediate phase in between the low-field antiferromagnet and the highfield ferromagnet. This extra phase is analyzed in details by Torrance and Tinkham [5], but is not included in the present analysis, since it is quite demanding to analyze by numerical methods. The calculated transition with field along $z$, is in good agreement with experiments, in the sense that the transition is predicted to occur in the narrow interval between the two experimental ones. The calculated critical field along the hard $y$ axis is $H_{c}^{y}=411 \mathrm{kOe}$ and the experimental value is $372 \mathrm{kOe}$. Similarly, the experimental critical field along the $x$ axis is

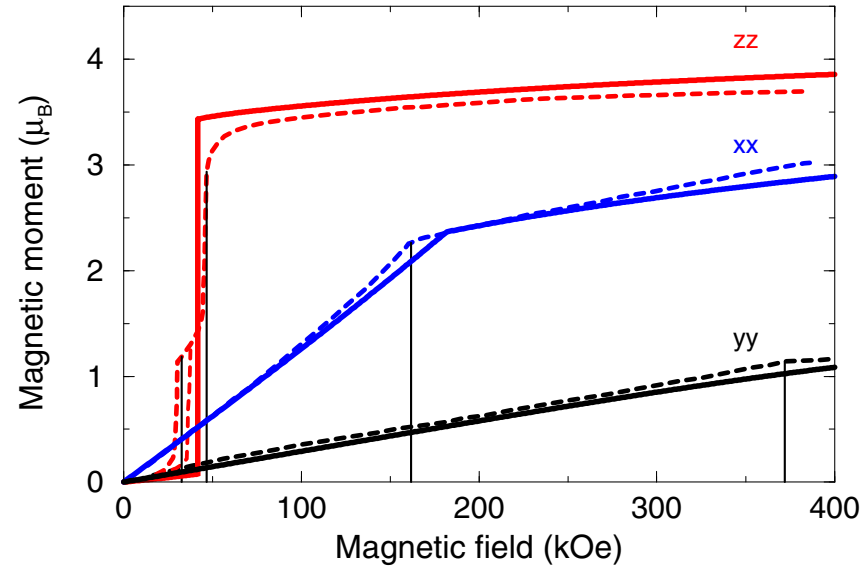

FIG. 3. Magnetization curves of $\mathrm{CoCl}_{2} \cdot 2 \mathrm{H}_{2} \mathrm{O}$ in the antiferromagnetic phase at $1.3 \mathrm{~K}$ measured by Mollymoto et al. [23]. The experimental results are shown by the dashed lines and the thin vertical lines indicate the critical fields. The colors red, blue, and black denote the $z z, x x$, and $y y$ components, respectively, and the solid lines are the calculated results.

$H_{c}^{x}=162 \mathrm{kOe}$, somewhat smaller than the theoretical value of $182 \mathrm{kOe}$. The phenomenological $H^{3}$-term considered by Mollymoto et al. [23], to arise from the field-induced mixing of the higher lying doublets into the ground state doublet, is accounted for in Fig. 3, when assuming that the additional contributions from the ${ }^{2} E$ term at about $2.3 \mathrm{eV}$ above ${ }^{4} F$ in the free $\mathrm{Co}^{++}$ion [11] and from other higher lying terms are negligible compared to the ${ }^{4} P$ contributions. The MF value of the critical field along the $x$ axis depends on $p$, where $p$ is the number of spins in the cluster basis, and we get $H_{c}^{x}=201.2,188.4,184.1$, and $181.8 \mathrm{kOe}$ for $p=1,2,3$, and 4 , respectively. Plotting $H_{c}^{x}$ as a function of $1 / p$ we get a straight line, and the extrapolated value for the infinite chain, $1 / p=0$, is found to be $H_{c}^{x}=175 \mathrm{kOe}$. Similarly, we get $H_{c}^{y}=386 \mathrm{kOe}$ when extrapolating to the infinite chain. The remaining minor differences between the infinite chain results and the experimental ones of $4 \%-8 \%$ may very well be due to that the MF approximation is still in use in the account for the interchain interactions.

\section{THE MAGNETIC EXCITATIONS}

The neutron scattering results for the spin waves energies determined in the antiferromagnetic phase of $\mathrm{CoCl}_{2} \cdot 2 \mathrm{D}_{2} \mathrm{O}$ at zero field below $7 \mathrm{~K}$ are presented in Fig. 4. The calculated results shown by the solid lines are derived using the fourspin chain basis. The antiferromagnetic ordering implies the presence of two spin-wave modes linearly polarized along either the $x$ or the $y$ axis, and the two spin wave branches are named according to their polarization. This naming only applies in the Brillouin zones around lattice points $h \vec{a}^{\star}+$ $k \vec{b}^{\star}+\ell \vec{c}^{\star}$, where $h+k$ is even, whereas the two polarizations are interchanged when $h+k$ is odd. The exchange parameters in Table III are mostly determined by the fitting of the dispersion relation in Fig. 4. In comparison with the previous fits to the spin wave energies [19,21], we have introduced one more parameter, the next-nearest neighbor coupling $J_{0}^{\prime}$ along 


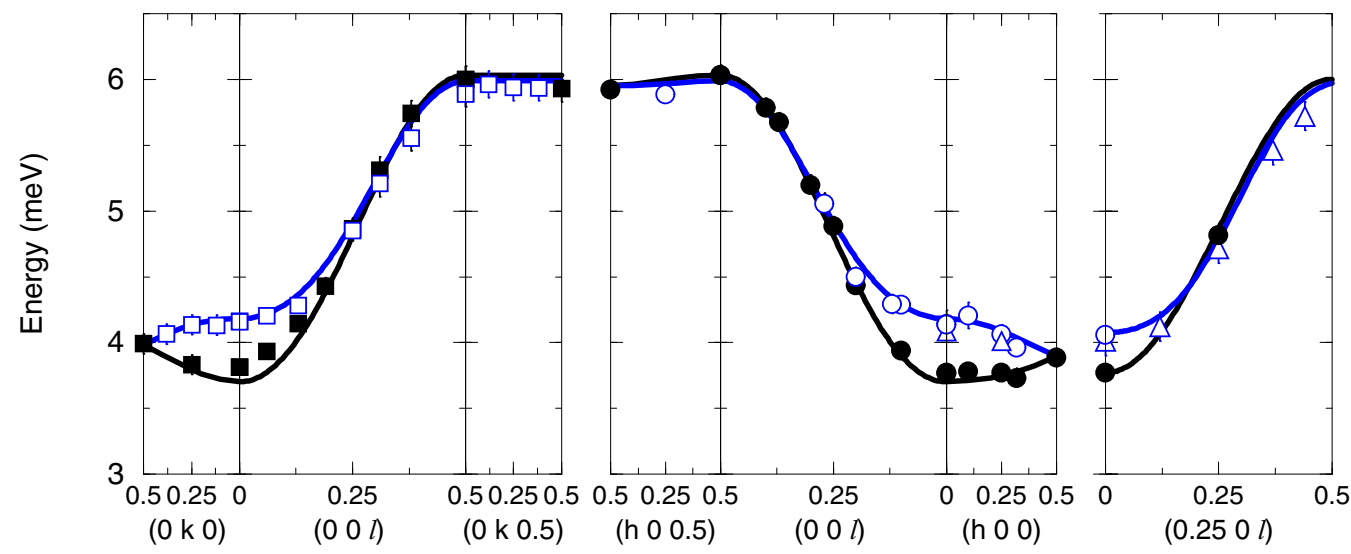

FIG. 4. The spin wave energies in the antiferromagnetic phase of $\mathrm{CoCl}_{2} \cdot 2 \mathrm{D}_{2} \mathrm{O}$ determined by neutron scattering at temperatures below $7 \mathrm{~K}$. The circles denote the results obtained by Montfrooij et al. [21]. The results reported in Refs. [19,20] are shown by squares and triangles, respectively. Open symbols refer to the $x$ mode and filled ones to the $y$ mode. The results of the fitting procedure to the excitation energies for the $x$ and $y$ modes are given by the blue and black lines, respectively.

the chains, and allowed $J_{1}$ and $J_{1}^{\prime}$ to be different. We have to add, that the antiferromagnetic spin waves are only weakly dependent on the difference between $J_{1}$ and $J_{1}^{\prime}$, and that $J_{1}^{\prime}$ is hardly distinguishable from the interaction $J_{1}^{\prime \prime}$ between ions at $\vec{r}_{i}-\vec{r}_{j}= \pm\left(\frac{1}{2} \vec{a}-\vec{c}\right) \pm \frac{1}{2} \vec{b}$.

Torrance and Tinkham have studied the excitation spectrum in $\mathrm{CoCl}_{2} \cdot 2 \mathrm{H}_{2} \mathrm{O}$ at zero wave vector by means of far-infrared transmission measurements [5]. In addition to the spin waves they observed a number of resonances, which they explained to be due to magnon bound states or, more specifically, to spin reversal of clusters of spins consisting of 2, 3, or more spins along the $c$ axis chains [4,5]. Clearly, these extra resonances do not appear in a simple RPA theory based on the single spins. However, considering instead a basis of 2 or more neighboring spins along the chain, the RPA theory is capable of accounting for the extra excitations. The more spins included in the clusters, the more resonances appear and the more accurate the theory becomes. Numerical analyses of cluster models with up to seven spins with $S($ eff $)=1 / 2$ indicate that the $S($ eff $)=3 / 2$ model with four spins in the clusters can be trusted with respect to its predictions for the lowest three pairs of excitations, and that it also gives a fair description of the fourth lowest excitation pair (the dashed lines in Fig. 5). The structure stays antiferromagnetic below the critical field $H_{c 1} \simeq 31.6 \mathrm{kOe}$, and it is ferromagnetic above $H_{c 2} \simeq 45 \mathrm{kOe}$. Figure 5 shows the comparison between experiments and theory in these two phases. The only noteworthy discrepancy found in this comparison is that the calculations show no sign of the fifth mode $\left(e_{5}\right.$ in the nomenclature of Torrance and Tinkham) above $H_{c 2}$. Applying the simpler $S$ (eff) $=1 / 2$ model, the $e_{5}$ mode does appear at about the right energies, when using clusters with six or seven spins. In between the two critical fields, the spins are ordered in a ferrimagnetic structure and show the resonances expected, as discussed by Torrance and Tinkham [5]. Their experiments also revealed a coupling between the magnetic excitations and an optical phonon at about $3.63 \mathrm{meV}$. Nicoli and Tinkham have estimated that this optical phonon mode is most likely due to the hydrogen ions in the water molecules [24]. In general, the replacement of $\mathrm{H}$ with
D only have weak influences on the magnetic properties, and in the actual case on the positions of the resonances. The only exception is that the hydrogen mode is shifted $10 \%$ down in energy to be around $3.3 \mathrm{meV}$ as measured recently by terahertz spectroscopy [25].

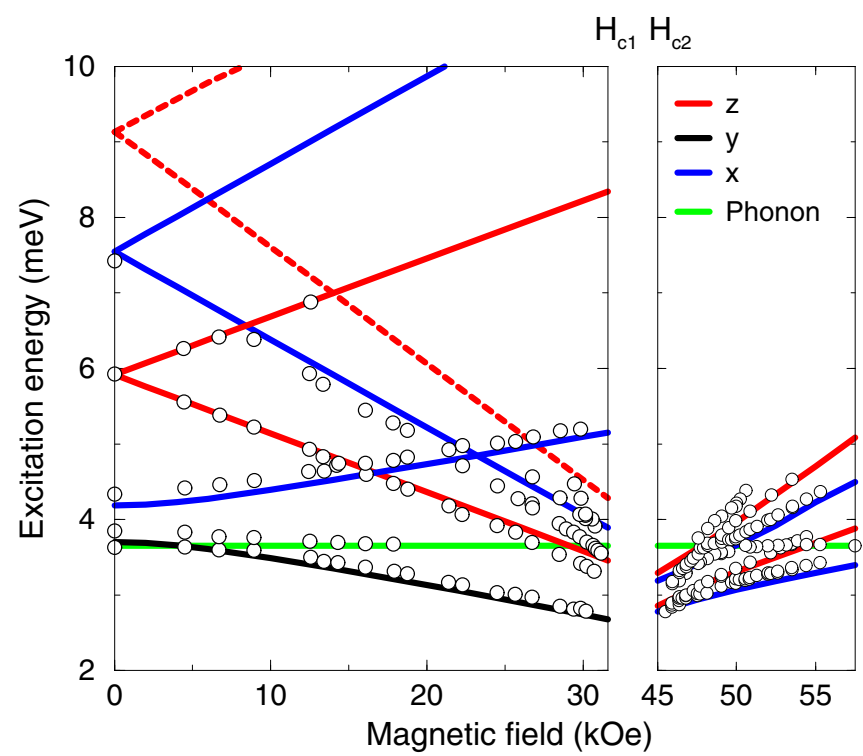

FIG. 5. The resonance frequencies in $\mathrm{CoCl}_{2} \cdot 2 \mathrm{H}_{2} \mathrm{O}$ at 1.6 and $6 \mathrm{~K}$ observed by Torrance and Tinkham in far-infrared transmission experiments [5]. The results are plotted against a field applied along the $b(z)$ axis. The excitation energies calculated using the four-spin cluster model are shown by the solid lines. The transverse-mode labels $x$ and $y$ are only strictly valid for the spin wave modes (the two lowest lying modes) at zero field. Else, all modes labeled by $x$ contain a minor elliptical $y$ component, and the $x$ component is also the dominant one in the $y$ mode at fields above $10 \mathrm{kOe}$. The green horizontal line indicates the optical phonon branch, which is observed because this phonon interact with the magnetic excitations as discussed in Refs. [5,24]. 


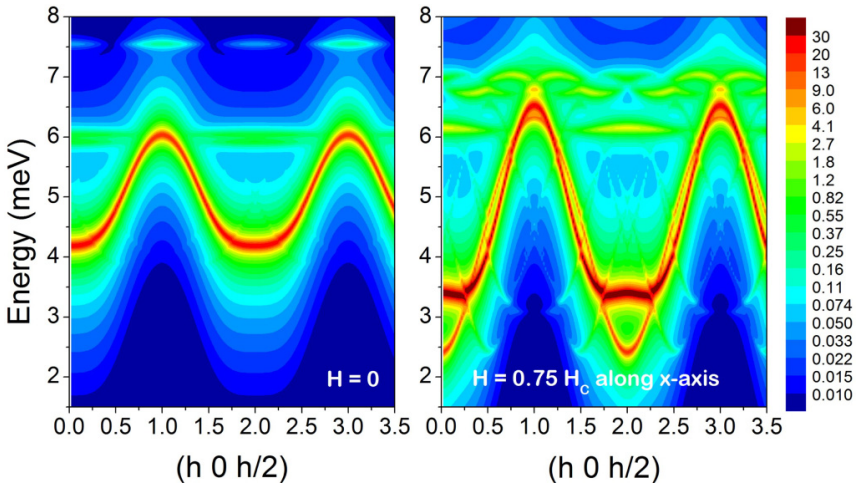

FIG. 6. The transverse correlation function at $1.3 \mathrm{~K}$ calculated along $Q=(h 0 h / 2)$ plotted on a logarithmic scale. The figure to the left shows the zero-field result, and the figure to the right is the calculated result at the $x$-axis field $H=0.75 H_{c}^{x}$, corresponding to $120 \mathrm{kOe}$ in the experiment and $137 \mathrm{kOe}$ in the model calculation.

Figure 6 shows contour plots of the transverse correlation function calculated along $(h 0 h / 2)$ at zero field and at $H=$ $0.75 H_{c}^{x}$ applied along the easy axis within the $a-c$ plane, i.e., the $x$ axis. The transverse correlation function is proportional to the neutron-scattering cross section, when neglecting the minor correction due to form factor variations. The logarithmic scale is used in order to make the weak chain-cluster excitations visible. The neutron scattering cross sections at zero field of the two- and three-spin flip excitations, at about 6 and $7.5 \mathrm{meV}$, are a factor of 10 or more smaller than that of the spin waves. They become more intense, and more dispersive, when approaching the critical field along the $x$ axis. However, the experimental conditions are difficult to handle when applying a large magnetic field, and the size/quality of the crystal is too poor to allow more than just the most intense spin waves to be observed (see next section).

The relatively small cross sections of the higher-order spin-flip excitations are in agreement with the results obtained by Shinkevich and Syljuåsen [2]. In order to make the higher-order excitations more visible, they propose to heat the system to temperatures close to the Néel temperature, at which temperatures it should be possible to observe low-energy transitions between the excited states. The present model calculations, particularly when using the $S($ eff $)=1 / 2$ model with six-seven spins in the chain clusters, are consistent in most details with the results derived from the theory of Shinkevich and Syljuåsen. The estimates they present for the case of $\mathrm{CoCl}_{2} \cdot 2 \mathrm{D}_{2} \mathrm{O}$ are probably a little too optimistic, since they assume the anisotropy parameter $j^{a}$ defined by Eq. (4) to be $j^{a}=0.21$, a factor of 2 larger than the value derived here. In the low temperature case, the possibility for observing the higher-order cluster spin excitations by neutrons has turned out to be most favored by a field applied along the $z$ direction [25].

\section{HIGH-FIELD NEUTRON SCATTERING EXPERIMENTS}

Figures 7 and 8 show additional results from the inelastic neutron scattering experiments presented in Ref. [1] employing the triple axis spectrometers RITA-II (SINQ, Switzerland). The inelastic scattering intensities observed at (030) as function of a field applied along the hard $y$ axis are shown in Fig. 7.

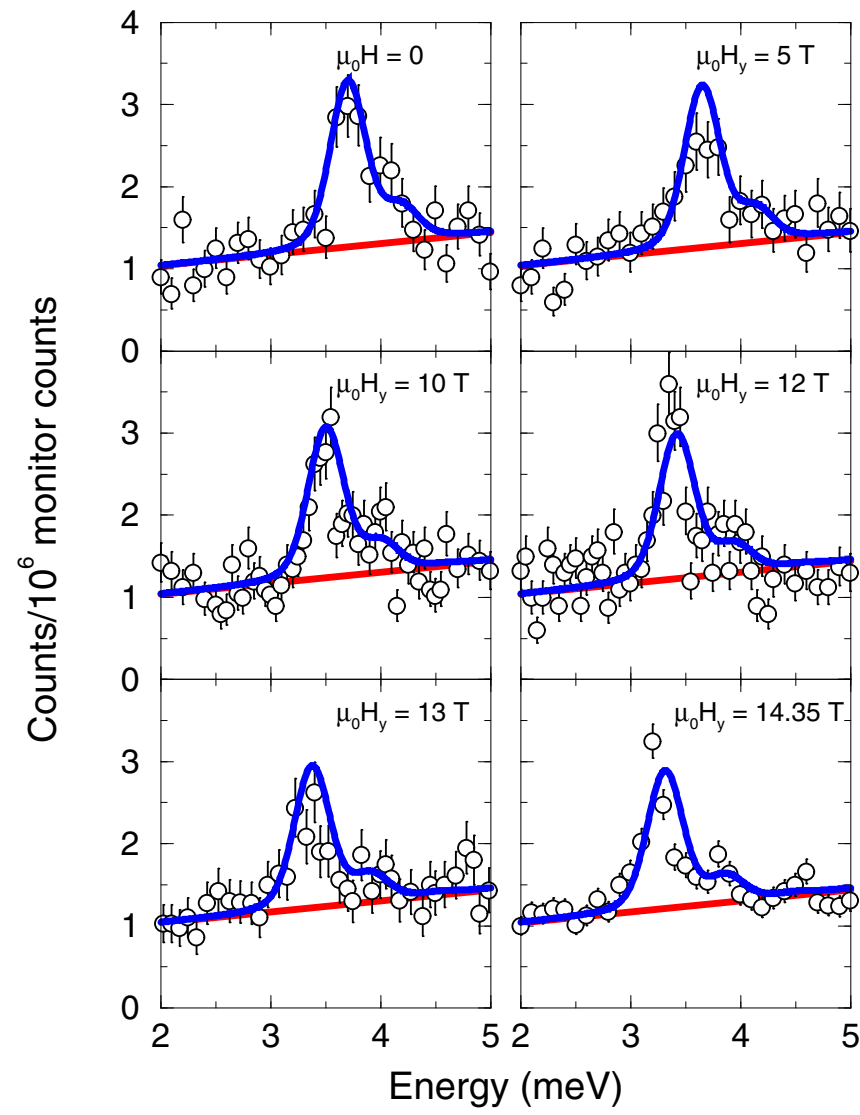

FIG. 7. Inelastic scans at $(030)$ in $\mathrm{CoCl}_{2} \cdot 2 \mathrm{D}_{2} \mathrm{O}$ at various fields along the hard $y$ axis at $1.5 \mathrm{~K}$. The circles are the experimental points and the blue lines are the results obtained when assuming the same experimental resolution and the same background, the red lines, in all cases. The experimental results nominally at $14.35 \mathrm{~T}$ are the average of those obtained at fields $14,14.2,14.4$, and $14.8 \mathrm{~T}$ reducing the scatter by a factor of 2 .

The calculated results are obtained from the four-spin cluster model. The large peak derives from the $x$ and the weak one from the $y$ polarized excitation. Notice that these polarizations, and hence also the distribution of intensities, are the opposite of that applying in Fig. 4. In spite of the large scatter in the experimental points, the agreement between the calculated and experimental results is sufficiently good to indicate that the theory predicts about the right relative intensities of the two spin wave modes.

Like the scans at (030), the scans at (211) with the field along the $x$ axis in Fig. 8 show a double peak structure. At this $\Gamma$ point and at fields $\leqslant 50 \mathrm{kOe}$, the lower one of the two spin wave modes at zero wave vector is the $x$-polarized mode, and it is the most intense one, since the intensity of the upper $y$-polarized mode is vanishingly small at zero field. $\left(g^{y} / g^{x}\right)^{2} \simeq 0.3$ and the angle between the scattering vector and the $y$ axis is small $\left(\sim 16^{\circ}\right)$. Applying the field the moments are rotated towards the $x$ axis. The $y$-polarized mode becomes elliptical polarized in the $y-z$ plane, giving rise to a strong enhancement of its cross section, whereas the creation of a static $x$ moment implies that the dynamic $x$-polarized mode is weaken in intensity. At the phase transition, the antiferromagnetically ordered $z$ component vanishes, and the moments become aligned along 


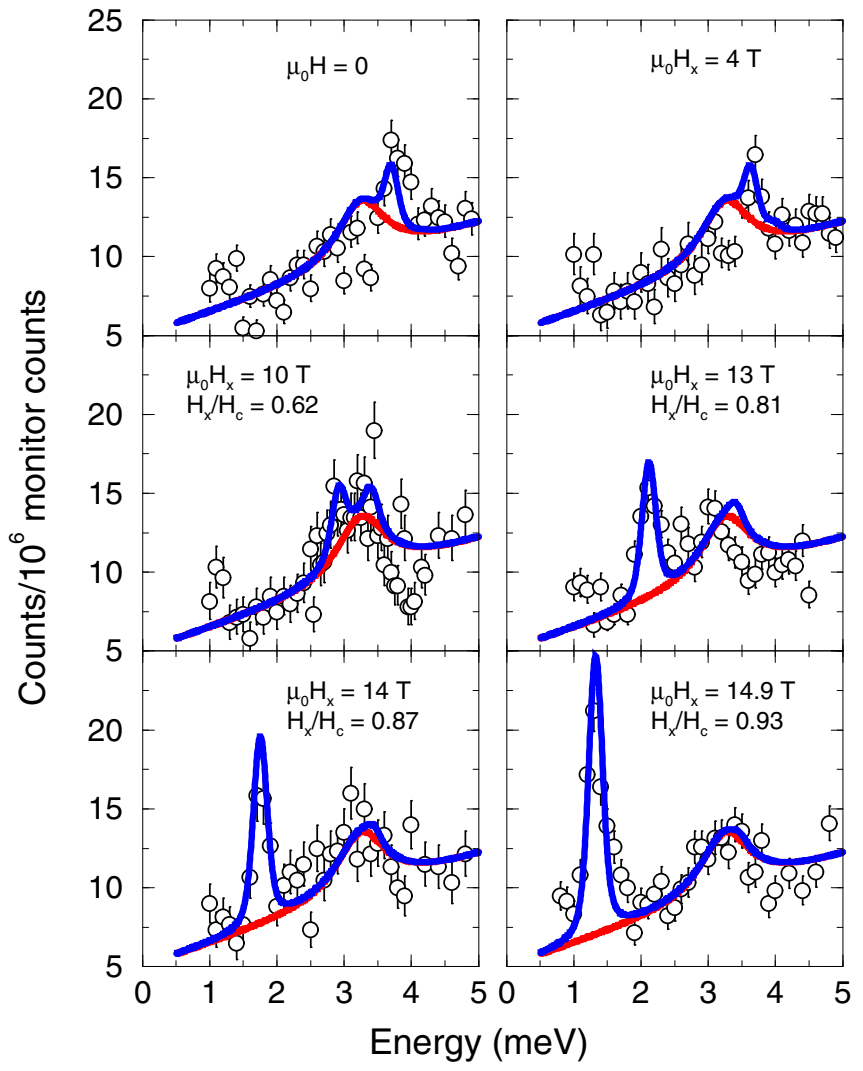

FIG. 8. Inelastic neutron scattering results obtained at (2 111$)$ in $\mathrm{CoCl}_{2} \cdot 2 \mathrm{D}_{2} \mathrm{O}$ at fields applied along the $x$ axis at $1.5 \mathrm{~K}$. The signatures are the same as used in Fig. 7. The calculations are performed at fields determined so that $H / H_{c}^{x}$ is the same as the experimental values.

the $x$ axis. The critical field in the $x$ direction derived from the neutron scattering experiments [1] is $160.5 \mathrm{kOe}$ close to the value of $162 \mathrm{kOe}$ determined by Mollymoto et al. [23]. Approaching the critical field from below, the intensity of the $x$-polarized mode vanishes, and it is no longer present above the critical field. At the field $\mu_{0} H=14.9$ T, i.e., at $H / H_{c}^{x}=0.93$, the intensity of the $x$ polarized mode is expected to be $30-40$ times smaller than the intensity of the $y-z$ polarized mode. The experiment at this field, presented in Fig. 8, shows a broad peak centered at about $3.3 \mathrm{meV}$ in addition to the narrow one due to the $y-z$ polarized mode at $1.4 \mathrm{meV}$. The integrated intensity of the broad peak is as large as that of the $y-z$ mode, implying that this peak is not the $x$-polarized mode but something else. If we assume that the broad peak is nonmagnetic and, hence, belongs to the background of the magnetic scattering, we get an acceptable description of all scans presented in Fig. 8. It is most likely that the nonmagnetic peak is the optical phonon mode detected at $3.65 \mathrm{meV}$ in the crystal with hydrogen [5], and shifted in energy to be about 3.3 $\mathrm{meV}$ in the deuterated crystal [25]. This mode may hybridize with the $x$ mode lying in the same energy range, whereas the $y-z$ polarized excitation does not seem to be much influenced by the phonon mode.

The $y-z$ polarized mode, which is present both above and below the transition, shows a soft mode behavior, i.e., the energy goes to zero, the intensity diverges, and the $y$ component of the elliptical polarization vanishes in the limit

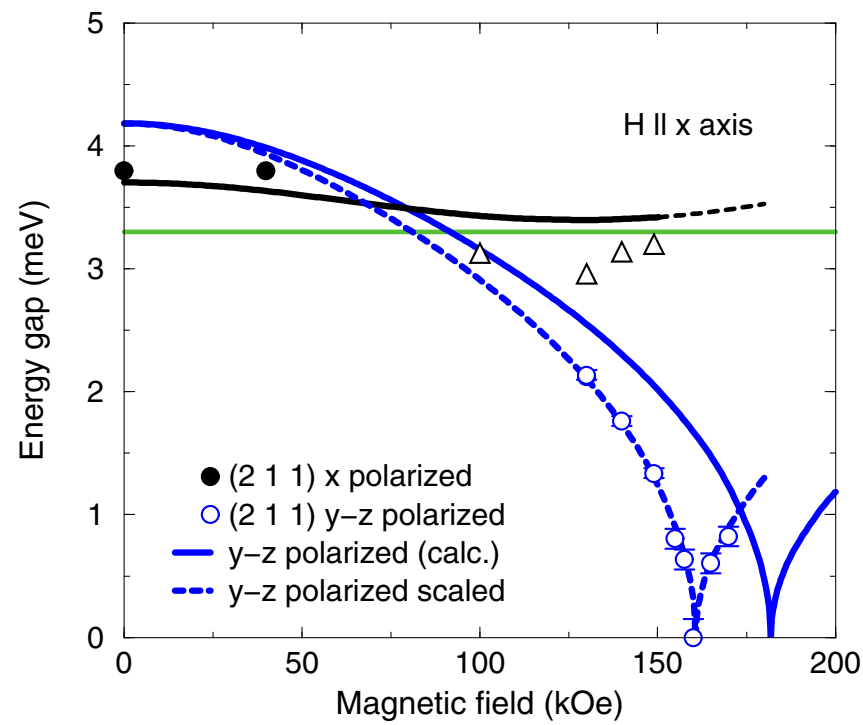

FIG. 9. The energy variation of the excitations at (211) in $\mathrm{CoCl}_{2} \cdot 2 \mathrm{D}_{2} \mathrm{O}$, when a field is applied along $x$. The triangles denote the positions of the extra peaks observed experimentally, which may derive from a combination of the $x$-polarized mode and an optical phonon mode. The position of the optical mode is shown by the thin green line. The cross section of the $x$-polarized mode becomes very small (dashed line) above $150 \mathrm{kOe}$. The dashed blue line is the calculated result scaled so that the critical field coincides with the experimental one, $H_{c}=160.5 \mathrm{kOe}$ [1].

of $H \rightarrow H_{c}^{x}$. The soft-mode behavior of this mode has been studied in detail by neutron experiments performed in the close neighborhood of the transition [1]. The excitation energies derived from the neutron scattering scans displayed in Ref. [1] and those derived from the scans in Fig. 8 are presented in Fig. 9. The four-spin cluster RPA model predicts that the energy of the soft spin-wave mode should be proportional to $\left|H-H_{c}^{x}\right|^{1 / 2}$ in a rather large interval around $H_{c}^{x}$, and, as shown by the good agreement between experiments and theory in Fig. 9, the experiments lead to the same conclusion [1].

\section{CONCLUSIONS}

The four-spin chain cluster model developed here gives an accurate account of most experiments addressing the magnetic properties of the anisotropic Co-chain system $\mathrm{CoCl}_{2} \cdot 2 \mathrm{H}_{2} \mathrm{O}$. This applies to previous experimental investigations as well as to the present determinations of the energy of the excited doublet and the behavior of the spin waves at high magnetic fields. One important conclusion drawn from our reanalysis of the spin waves is that the exchange interaction $J_{0}^{\prime}$ between next-nearest neighbors on the $c$-axis chains is significant. This interaction is going to modify the analytic results derived for the simple one-dimensional Ising model $[10,26]$.

The $S($ eff $)=3 / 2$ model is quite complex, since the ${ }^{4} P$ term has to be included in order to get the right values for the wave functions and for the splitting between the two doublets. Fortunately, the simpler $S($ eff $)=1 / 2$ model determined by the $g$ factors in Table II and the effective exchange parameters given by Eq. (3) in combination with Table III, may be an acceptable approximation in many investigations. For instance, 
the $S($ eff $)=1 / 2$ model with clusters of 6 spins along the chains predicts spin-wave energies that deviate, at maximum, by only 4\% from that shown in Fig. 4.

The Ising character of the exchange interaction in $\mathrm{CoCl}_{2} \cdot 2 \mathrm{H}_{2} \mathrm{O}$ and in the similar chain system $\mathrm{CoNb}_{2} \mathrm{O}_{6}$ is comparable [10]. The same applies to the magnitude of the nearest-neighbor chain interaction $J_{0}$, however, the Néel temperature, reflecting the exchange interactions between spins on neighboring chains, is about six times higher in $\mathrm{CoCl}_{2} \cdot 2 \mathrm{H}_{2} \mathrm{O}$ than in $\mathrm{CoNb}_{2} \mathrm{O}_{6}$. In the ordered phase, the interchain interactions perturb the one-dimensional chain system with an effective longitudinal field. In comparison with $\mathrm{CoNb}_{2} \mathrm{O}_{6}$, this longitudinal field is relatively strong in $\mathrm{CoCl}_{2} \cdot 2 \mathrm{H}_{2} \mathrm{O}$ making the low-dimensionality features less pronounced. Actually, the only one-dimensionality marker left in $\mathrm{CoCl}_{2} \cdot 2 \mathrm{H}_{2} \mathrm{O}$ seems to be the bound magnon states due to the spin reversal of two or more neighboring spins along the chains, which were detected by far-infrared transmission and explained by Torrance and Tinkham [5]. However, it is worth to notice that the intrachain interaction is strong enough to make the simple MF/RPA theory untrustworthy. The MF approximation, where the basis is the single spins, overestimates the Néel temperature by $70 \%$ and the critical fields by about $30 \%$.

In agreement with the theory of Shinkevich and Syljuåsen [2], the present four-spin cluster calculations show that the neutron cross sections of any other excitations than the spin waves are small. The behavior of the magnetic excitations predicted by the cluster model calculations are in close agreement with a great variation of experiments, and all its more general predictions, also beyond what may be observed experimentally at present, are in substantial agreement with those derived from the theory of Shinkevich and Syljuåsen. This consistency allows us to conclude that the elementary chain excitations in $\mathrm{CoCl}_{2} \cdot 2 \mathrm{H}_{2} \mathrm{O}$ do not derive from the single domain walls, as assumed by Kyriakidis and Loss [3], but from the bound pairs of domain walls considered by Shinkevich and Syljuåsen [2].

The effective longitudinal field is canceled just at the critical transverse field $H_{c}$, where the antiferromagnetic order is destroyed, implying that free oscillations of the single domain walls become a possibility just at this field. The excitations spectrum close to the quantum phase transition at the critical field $H_{c}^{x}$ in the zero temperature limit was examined carefully in Ref. [1] and no sign of anything else but the spin waves were observed. The energy of the critical spin-wave mode is found to be proportional to $\left|H-H_{c}^{x}\right|^{1 / 2}$ on both sides of the transition. This soft mode behavior is similar with that shown by the three-dimensional Ising ferromagnet $\mathrm{LiHoF}_{4}$ in a transverse field [27], except that the closure of the energy gap is interrupted by the hyperfine interaction in the case of $\mathrm{LiHoF}_{4}$, whereas the softening is complete in $\mathrm{CoCl}_{2} \cdot 2 \mathrm{D}_{2} \mathrm{O}$ [1].

\section{ACKNOWLEDGMENTS}

The authors would like to thank Kim Lefmann and Niels Bech Christensen for help with the experiments and stimulating discussions. The development of the model for the Co ions have benefited much from discussions with Martin Rotter and Manh Duc Le. We thank Turi Schäffer, Sonja Holm, and Jonathan Taylor for taking part in some of the experiments.
The project was supported by the Danish Agency for Science and Higher Education through DANSCATT.

\section{APPENDIX: THE SUSCEPTIBILITY BASED ON THE ISING CHAIN}

The susceptibility tensor determining the heavy solid lines in Fig. 2 is based on the exact result for the susceptibility of the 1D Ising chain. To leading order, the system is considered to be an $S=1 / 2$ Ising system, and all perturbations of this system are assumed to be accounted for by the MF approximation. By far, the strongest interaction between the magnetic moments in this system is the nearest neighbor exchange interaction, $J_{0}$, between the $b(z)$ components of the spins. Introducing the $z z$ component of the local spin-spin susceptibility tensor, $\chi_{S_{z} S_{z}}^{0}=$ $\left|\left\langle S_{z}\right\rangle\right|^{2} / k_{B} T$, as determined by the matrix element of $S_{z}$ within the ground-state doublet, the exact susceptibility for the 1D Ising chain is

$$
\chi_{S_{\alpha} S_{\alpha}}^{1 \mathrm{D}}=\chi_{S_{\alpha} S_{\alpha}}^{0} \exp \left(2 J_{0} \chi_{S_{\alpha} S_{\alpha}}^{0}\right)
$$

with $S_{\alpha}=S_{z}$. The first perturbation of this result, that the actual spin-spin susceptibility is modified by the excited doublet, is simply accounted for by replacing the doublet susceptibility $\chi_{S_{z} S_{z}}^{0}$ considered above, with the one determined by the total doublet-doublet system of the single Co ions. When all other interactions than $J_{0}$ are neglected, the system becomes an $X Y Z$-Heisenberg not an Ising chain. Exact results exist for the $X Y Z$-Heisenberg chain (see, for instance, Ref. [28]), but are difficult to apply. Since the $x$ and $y$ components are weak we assume, in accordance with the MF approximation, that the three components may be considered to be independent of each other, and, in that case, that all three diagonal susceptibility components are determined by Eq. (A1), with $\alpha=x, y$, or $z$. The spin-spin exchange interaction also affects the orbital susceptibility components, and based on the MF approximation, the orbital 1D chain susceptibilities are

$$
\chi_{L_{\alpha} S_{\alpha}}^{1 \mathrm{D}}=\chi_{L_{\alpha} S_{\alpha}}^{0} \exp \left(2 J_{0} \chi_{S_{\alpha} S_{\alpha}}^{0}\right)
$$

and

$$
\chi_{L_{\alpha} L_{\alpha}}^{1 \mathrm{D}}=\chi_{L_{\alpha} L_{\alpha}}^{0}+2 J_{0} \chi_{L_{\alpha} S_{\alpha}}^{0} \chi_{L_{\alpha} S_{\alpha}}^{1 \mathrm{D}} .
$$

The last step is to introduce the remaining interactions $J(0)-$ $2 J_{0}$, and applying the MF approximation once again, the final results are

$$
\begin{aligned}
& \chi_{S_{\alpha} S_{\alpha}}=\chi_{S_{\alpha} S_{\alpha}}^{1 \mathrm{D}} / D ; \quad D=1-\left[J(0)-2 J_{0}\right] \chi_{S_{\alpha} S_{\alpha}}^{1 \mathrm{D}}, \\
& \chi_{L_{\alpha} S_{\alpha}}=\chi_{L_{\alpha} S_{\alpha}}^{1 \mathrm{D}} / D, \\
& \chi_{L_{\alpha} L_{\alpha}}=\chi_{L_{\alpha} L_{\alpha}}^{1 \mathrm{D}}+\left[J(0)-2 J_{0}\right] \chi_{L_{\alpha} S_{\alpha}}^{1 \mathrm{D}} \chi_{L_{\alpha} S_{\alpha}},
\end{aligned}
$$

and the total magnetic susceptibility tensor is given by

$$
\chi_{\alpha \alpha}=\mu_{B}^{2}\left(\chi_{L_{\alpha} L_{\alpha}}+4 \chi_{L_{\alpha} S_{\alpha}}+4 \chi_{S_{\alpha} S_{\alpha}}\right) .
$$


[1] J. Larsen, T. K. Schäffer, U. B. Hansen, S. L. Holm, S. R. Ahl, R. Toft-Petersen, J. Taylor, G. Ehlers, J. Jensen, H. M. Rønnow, K. Lefmann, and N. B. Christensen, Phys. Rev. B 96, 174424 (2017).

[2] S. Shinkevich and O. F. Syljuåsen, Phys. Rev. B 85, 104408 (2012).

[3] J. Kyriakidis and D. Loss, Phys. Rev. B 58, 5568 (1998).

[4] J. B. Torrance Jr., and M. Tinkham, Phys. Rev. 187, 587 (1969).

[5] J. B. Torrance Jr., and M. Tinkham, Phys. Rev. 187, 595 (1969).

[6] S. Sachdev, Quantum Phase Transitions (Cambridge University Press, Cambridge, 1999).

[7] D. E. Cox, B. C. Frazer, and G. Shirane, Phys. Lett. 17, 103 (1965).

[8] A. Narath, Phys. Rev. 136, A766 (1964).

[9] A. Narath, Phys. Rev. 140, A552 (1965).

[10] R. Coldea, D. A. Tennant, E. M. Wheeler, E. Wawrzynska, D. Prabhakaran, M. Telling, K. Habicht, P. Smeibidl, and K. Kiefer, Science 327, 177 (2010).

[11] R. A. Cowley, W. J. L. Buyers, C. Stock, Z. Yamani, C. Frost, J. W. Taylor, and D. Prabhakaran, Phys. Rev. B 88, 205117 (2013).

[12] A. Abragam and M. H. L. Pryce, Proc. R. Soc. London A 206, 173 (1951).

[13] A. Abragam and B. Bleaney, Electron Paramagnetic Resonance of Transition Ions (Oxford University Press, Oxford, 1970).

[14] M. T. Hutchings, Solid State Phys. 16, 227 (1964).
[15] J. Jensen and A. R. Mackintosh, Rare Earth Magnetism: Structures and Excitations (Clarendon Press, Oxford, 1991), http://www.nbi.ku.dk/page40667.htm.

[16] J. Jensen, Phys. Rev. B 79, 014406 (2009).

[17] J. Jensen, Phys. Rev. B 84, 104405 (2011).

[18] M. Rotter, M. D. Le, A. T. Boothroyd, and J. A. Blanco, J. Phys.: Condens. Matter 24, 213201 (2012); http://www.mcphase.de/

[19] J. K. Kjems, J. Als-Nielsen, and H. Fogedby, Phys. Rev. B 12, 5190 (1975).

[20] N. B. Christensen, K. Lefmann, I. Johannsen, and O. Jørgensen, Physica B (Amsterdam) 276-278, 784 (2000).

[21] W. Montfrooij, G. E. Granroth, D. G. Mandrus, and S. E. Nagler, Phys. Rev. B 64, 134426 (2001).

[22] J. Larsen, Master thesis, University of Copenhagen, 2009 and J. Larsen, Ph.D. thesis, Technical University of Denmark, 2013.

[23] H. Mollymoto, M. Motokawa, and M. Date, J. Phys. Soc. Jpn. 49, 108 (1980).

[24] D. F. Nicoli and M. Tinkham, Phys. Rev. B 9, 3126 (1974).

[25] U. B. Hansen et al. (unpublished).

[26] J. A. Kjäll, F. Pollmann, and J. E. Moore, Phys. Rev. B 83, 020407(R) (2011).

[27] H. M. Rønnow, R. Parthasarathy, J. Jensen, G. Aeppli, T. F. Rosenbaum, and D. F. McMorrow, Science 308, 389 (2005); H. M. Rønnow, J. Jensen, R. Parthasarathy, G. Aeppli, T. F. Rosenbaum, D. F. McMorrow, and C. Kraemer, Phys. Rev. B 75, 054426 (2007).

[28] M. Takahashi, Thermodynamics of One-Dimensional Solvable Models (Cambridge University Press, Cambridge, 1999). 